\title{
Light, Lasers, and the Nobel Prize
}

\section{John M. Dudley}

The Year 2020 represents 60 years since the first successful operation of the laser. This anniversary provides an ideal occasion to reflect on the myriad ways that lasers have revolutionized society, and to consider the many new areas of research that continue to drive photonics in unexpected directions. Yet at the same time as we consider these exciting future perspectives, it is also interesting to see how the development of the laser traces a path that intertwines basic and applied science, and intersects with the recognition of many of the pioneers of optics through the Nobel Prize. Of course, an exhaustive history of such a rich topic cannot be given in a short Perspective, but it is perhaps possible to describe some of the key highlights.

A good point to begin any historical discussion of the laser is the second half of $19^{\text {th }}$ century, and the study of the emission properties of hot objects and the measurements of the characteristic spectrum of black body radiation. In fact, it is not widely appreciated that these studies were not initially motivated by questions of fundamental scientific curiosity, but were rather stimulated by a very practical and economic problem. ${ }^{1}$ In particular, the city of Berlin at the time was choosing between gas and electric lighting, essentially the same problem as we have had in recent years in switching from incandescent and fluorescent lights to LEDs. Naturally, when making such a decision, standardizing the spectral content of the different light sources was a critical first step, and it was this that drove experiments to measure precision radiation curves of sources at different temperatures. Theoretical work by Wien was able to connect the peak emission wavelength and the source temperature, but explaining the shape of the emission curve was only possible with the introduction of energy quantization by Max Planck in 1900.

Although the initial measurements of blackbody radiation may have had a strong industrial link, the scientific environment of the time was clearly focused on understanding the deep and fundamental questions concerning the nature of light-matter interactions. Indeed, the Nobel Prize was first awarded only in 1901, and the importance of studying the nature of light was quickly recognized with prizes to Lorentz and Zeeman (1902), Wien (1911), and Planck himself (1918). ${ }^{2}$ It was in 1905 that Albert Einstein revolutionized physics with his four celebrated Annus Mirabilis papers. It is perhaps fitting that the first of these concerned the very nature of light itself, where he applied the concept of light quantization to explain the photoelectric effect. ${ }^{3}$ In fact, when Einstein was awarded the Nobel Prize (in 1921), it was only this particular contribution that was highlighted in his citation - "for services to Theoretical Physics, and especially for his discovery of the law of the photoelectric effect."

It was in 1917 when Einstein made a key contribution to the laser through his prediction of the process of Stimulated Emission. ${ }^{4}$ As well as developing what is now the familiar rate equation theory of emission and absorption, Einstein's insight led him to realize that stimulated emission would be associated with the emitted and incident photon

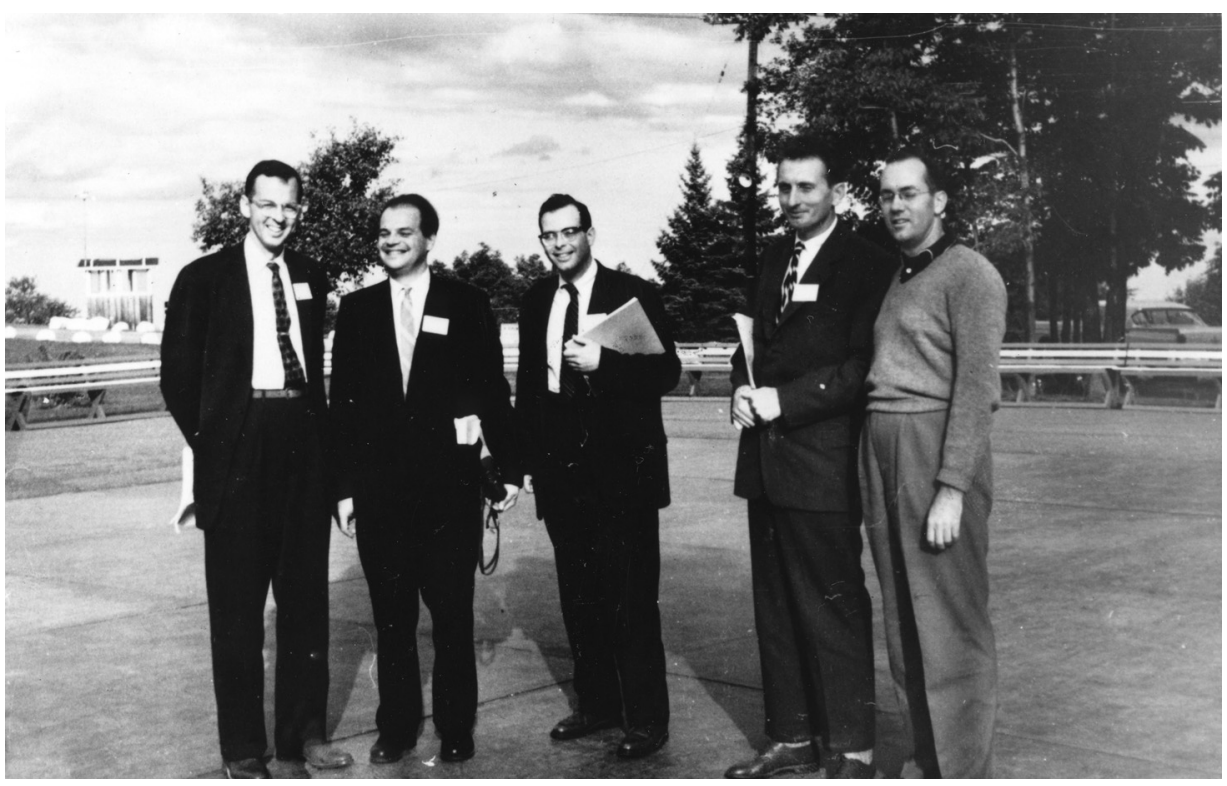

Fig. 1 (Left to right) James P. Gordon, Nikolai Basov, Herbert Zeiger, Alexander Prokhorov and Charles Hard Townes at the First Quantum Electronics Conference, Shawanga Lodge, September 14-16, 1959. Photo courtesy of The Regents of the University of California, Lawrence Berkeley National Laboratory.

(C) The Author. Published by SPIE and CLP under a Creative Commons Attribution 4.0 Unported License. Distribution or reproduction of this work in whole or in part requires full attribution of the original publication, including its DOI. [DOI: 10.1117/1 AP.2.5.050501] 
Physics

1902 Lorentz and Zeeman

The Zeeman Effect, Electron Oscillator Model

Physiology or Medicine

1903 Finsen

Phototherapy - use of UV light to treat Lupus

Physics

1907 Michelson

The Michelson Interferometer \& Precision Measurements

Physics

1908 Lippmann

Colour Photography based on Interference

Physiology or Medicine

Gullstrand

Description of the Refractive Optics of the Eye

Physics

1912 Dalén

Solar-based regulator for buoys and lighthouses

Physics

1918 Planck

Energy Quanta

Physics

1919 Stark

The Stark Effect

Physics

Einstein

Photoelectric Effect \& services to theoretical physics

Physics

1922 Bohr

Atomic Structure and the nature of radiation

Physics

1923 Millikan

Elementary Charge and the Photoelectric Effect

Physics

1927 Compton

The Compton Effect

Physics

1930 Raman

Raman scattering

Physics

1932 Heisenberg

Creation of Quantum Mechanics

Physics

1933 Schrodinger and Dirac

New Productive Forms of Atomic Theory

Physics

1945 Pauli

Pauli Exclusion Principle

Physics

1953 Zernike

Phase Contrast Microscope

Physics

1954 Born

Statistical Interpretation of the Wavefunction

Physics

1955 Lamb

Fine structure of the H Spectrum (Lamb Shift, QED)
Physics

1964 Townes, Basov, and Prokhorov

Maser-Laser Principle

Physics

1966 Kastle

Precision studies of optical resonances

Physiology or Medicine

1967 Granit, Hartline, and Wald

Physiological and chemical visual processes in the eye

Chemistry

1967 Eigen, Norrish, and Porter

Flashlamp Pump-Probe Studies of Chemical Reactions ( $\mu \mathrm{s}$ )

Physics

1971 Gabor

Holography

Physics

1981 Bloembergen and Schawlow

Laser Spectroscopy

Physiology and Medicine

1981 Hubel and Wiesel

Information Processing in the Visual System

Physics

1989 Ramsey, Dehmelt, and Paul

Atomic Clocks, the lon Trap

Physics

1997 Chu, Cohen-Tannoudji, and Phillips

Laser Cooling and Trapping

Chemistry

1999 Zewail

Femtochemistry

Physics

2000 Alferov and Kroemer

Optoelectronics, Semiconductor Heterostructures

Physics

2001 Cornell, Ketterle, and Wieman

Bose Einstein Condensation

Physics

2005 Glauber, Hall, and Haensch

Quantum Optics, Spectroscopy, Optical Frequency Comb

Chemistry

2008 Shimomura, Chalfie, and Tsien

Green Fluorescent Protein GFP

Physics

2009 Kao, Boyle, and Smith

Optical Fiber Communications ; Imaging and the CCD

Physics

2012 Haroche and Wineland

Individual Quantum Systems

Physics

2014 Akasaki, Amano, and Nakamura

The Blue LED and Energy-Saving White Light Sources

Chemistry

2014 Betzig, Hell, and Moerner

Super-resolution microscopy

Physics

2018 Ashkin, Mourou, and Strickland

Optical Tweezers \& Biophotonics

Chirped Pulse Amplification

Fig. 2 A selection of Nobel Prizes related to light, masers and lasers, and applications. The descriptions are highly abridged from the formal citation, and this is of course only a partial list. Many other Nobel Prizes have involved key areas of light science. In particular, some physics Nobel Prizes in astronomy and cosmology include laser instrumentation as central components e.g. gravitational wave detection and laser interferometery (Nobel 2017) and the observation of black holes building on laser guide star adaptive optics (Nobel 2020). A printable poster version is included as Supplementary Material. 
possessing the same direction. It is this directionality characteristic that provides the basis of amplification, and whilst Einstein did not foresee any form of practical laser device, his 1917 paper is nonetheless the foundation of everything that has followed since.

Building on these ideas, researchers extended both theory and experiment of light-matter interactions during the following decades, leading to the development of the concepts such as pumping and resonators, and ultimately the first demonstration of the maser in 1953 by Charles Townes and his PhD student Jim Gordon. With Arthur Schawlow, in 1958 Townes wrote a theoretical paper extending the maser concept into the visible spectrum, although they had yet to build an experimental prototype. ${ }^{5}$ These results established an entirely new field of "quantum electronics" and in 1959 Townes organized the first international conference (see Fig. 1) in the field where one of the important goals was to work towards extending the maser to optical wavelengths. ${ }^{6}$ Ted Maiman attended this conference, yet as he writes in his memoirs, he made a conscious decision to avoid the complex yet elegant systems that were being widely discussed, and to focus instead on practical simplicity. ${ }^{7}$ Of course, there was never any guarantee that Maiman's approach would work out, but on the 16 May 1960, while working at Hughes Research Laboratories, he observed pulsed laser oscillation at $694.3 \mathrm{~nm}$, building on his idea that flashlamp pumping of ruby would allow for a dynamic population inversion sufficient to reach threshold.

The pioneering work on both masers and lasers was recognized in 1964 with the award of the Nobel Prize to Charles Townes, Nicolay Basov, and Aleksandr Prokhorov "for fundamental work in the field of quantum electronics, which has led to the construction of oscillators and amplifiers based on the maser-laser principle." In fact, although many laser histories tend to focus mainly on the work performed in the USA, Basov and Prokhorov at the Lebedev Institute in Moscow were simultaneously and independently covering the same ground with their own theory and experiments. Two years later in 1966, Albert Kastler received the Nobel Prize for his work on optical pumping techniques, and later Bloembergen and Schawlow shared the 1981 Nobel Prize for laser applications in spectroscopy.

Maiman, despite being the first to see laser emission, never won the Nobel Prize, and neither did Jim Gordon. Whilst it is natural to consider these omissions as major oversights by the Nobel Committee, the available Nobel Prize archives ${ }^{8}$ reveal that the lack of any Nobel recognition for Maiman and Gordon may simply be linked to the fact that they were not strongly supported by the broader physics community at the time. In particular, starting as early as 1958, Charles Townes had been nominated 75 times for the Nobel Prize, including 29 nominations for the year in which he won. In contrast, based on what we know of the nomination archives (which are accessible until 1966), Gordon was nominated only once in 1963 and Maiman only once in 1964. This said, as far as Jim Gordon is concerned, Charles Townes certainly recognized the role he had played, and in 2014 he explicitly stated that "Jim didn't get the Nobel Prize with me, presumably because he was a student when the maser first worked, but I think he deserved it." 9

There were of course many other eminent scientists involved in the early years of laser physics, and some excellent personal and historical accounts are available. ${ }^{5,7,10,11}$ Lasers have also been recognized either directly or indirectly in many other Nobel Prizes as well (not just physics). Figure 2 lists a selection of Nobel Prizes related to the physics of light science and applications, before and after the invention of the laser and it is highly recommended to explore the Nobel Prize website to learn more.

Considering the history of the laser is an opportunity to think about many broader issues of science, and particularly the relationship between basic research and technology transfer. The laser is an ideal subject with which to explain the tremendous economic and societal benefits that can arise from basic curiosity-driven scientific research. With all the advances in photonics that continue to be made in many different areas, it is likely that laser-related science will continue to be recognized by Nobel Prizes in the future, and will continue to create revolutions in our lives.

\section{References}

1. H. Kubbinga, "A tribute to Max Planck," Europhys. News 49(4), 27-33 (2018).

2. The Nobel Prize in Physics has been awarded 113 times to 213 Nobel Laureates between 1901 and 2019. A full listing of laureates, as well as transcripts of the Presentation Speeches which provide important contextual information, can be seen at www .nobelprize.org.

3. A. Einsten, "Über einen die Erzeugung und Verwandlung des Lichtes betreffenden heuristischen Gesichtspunkt," Ann. Phys. 322(6), 132-148 (1905).

4. A. Einstein, "Zur Quantentheorie der Strahlung," Phys. Z. 18, 121-128 (1917).

5. C. H. Townes, How the Laser Happened: Adventures of a Scientist, Oxford University Press (1999)

6. The Shawanga Lodge Conference on Quantum ElectronicsResonance Phenomena 14-16 September 1959, Bloomingburg, New York. See also Physics Today 12(5), 72 (1959).

7. T. H. Maiman, The Laser Inventor: Memoirs of Theodore H. Maiman, Springer (2018)

8. Nomination Archive. NobelPrize.org. Nobel Media AB 2020.

9. "A historical perspective on the maser and laser," presented by Charles Townes, University of California, Berkeley, USA, and Tony Heinz, Columbia Univ., USA, The James P Gordon Symposium during CLEO 2014 in San Jose, California, USA.

10. J. Hecht, Beam: The Race to Make the Laser. Oxford University Press (2005). See also J. Hecht, "Beam: The Race to Make the Laser," Optics and Photonics News, 16(7), 24-29 (2005).

11. D. F. Nelson, R. J. Collins, and W. Kaiser, "Bell Labs and the ruby laser," Phys. Today 63(1), 40-45 (2010).

John Dudley is Professor of Physics at the Université BourgogneFranche-Comté and the CNRS Institute FEMTO-ST in Besancon, France. He initiated and chaired the UN International Year of Light \& Light-based Technologies 2015, and also chaired the follow-up annual UNESCO International Day of Light which is celebrated annually on the 16 May, the anniversary of the date in 1960 when Ted Maiman first observed laser oscillation. He is a Fellow of OSA, the IEEE, the IOP, and SPIE, and has won numerous awards, including the Médaille d'Argent of the French CNRS, the Harold E. Edgerton Award of SPIE, and the R. W. Wood Prize of OSA. 